\title{
Factores de riesgo para la presentación de bacteriuria en gatos con enfermedad del tracto urinario inferior: un análisis retrospectivo de 102 casos (2008 - 2015)
}

\author{
Risk factors for the presentation of bacteriuria in cats with lower urinary tract \\ disease: a retrospective analysis of 102 cases $(2008-2015)$
}

\author{
Christian Jordán D. ${ }^{1}$, Siever Morales-Cauti ${ }^{1,2,4}$, Alicia Rubio V. ${ }^{1}$, \\ Manuel Barrios-Arpi ${ }^{3}$, Eglinton Villacaqui-Ayllón ${ }^{1}$
}

\section{Resumen}

\begin{abstract}
El objetivo de este estudio retrospectivo fue describir los resultados de aislados bacterianos y determinar los factores de riesgo involucrados en la presentación de bacteriuria en gatos con enfermedad del tracto urinario inferior (FLUTD). Se recolectaron 102 historias clínicas de pacientes felinos a los cuales se les realizó análisis de orina y urocultivo. Se consideraron las variables edad, sexo, estado reproductivo, raza, peso, estación del año, color de orina, olor de orina, densidad urinaria, $\mathrm{pH}$, glucosa, proteínas, sangre, leucocitos, eritrocitos, cristales y tipo de cristales. En el 60.8\% (62/102) de las muestras se llegó a aislar bacterias en orina, entre ellas E. coli 40.3\% (25/62), Klebsiella sp 12.9\% (8/62), Staphylococcus sp 12.9\% (8/62), Proteus sp 8.1\% (5/62), Enterobacter sp $8.1 \%$ (5/62) y Staphylococcus epidermidis 6.4\% (4/62). La edad, densidad urinaria y presencia de sangre fue estadísticamente significativa. Además, se determinó que, la densidad urinaria, el color rojo de la orina, la presencia de leucocitos y de cristales representan factores de riesgo $(\mathrm{p}<0.05)$ para la ocurrencia de bacteriuria.
\end{abstract}

Palabras clave: gatos; urocultivo; ITU; bacteriuria; FLUTD

\section{Abstract}

The aim of this retrospective study was to describe the results of bacterial isolates and to determine the risk factors involved in the presentation of bacteriuria in cats with lower urinary tract disease (FLUTD). Clinical records of feline patients $(n=102)$ who

${ }^{1}$ Carrera de Medicina Veterinaria y Zootecnia, Universidad Cientifica del Sur, Lima, Perú

${ }^{2}$ Laboratorio de Microbiología y Parasitología Veterinaria, Facultad de Medicina Veterinaria, Universidad Nacional Mayor de San Marcos, Lima, Perú

${ }^{3}$ Laboratorio de Fisiología Animal, Facultad de Medicina Veterinaria, Universidad Nacional Mayor de San Marcos, Lima, Perú

${ }^{4}$ E-mail: sieverm@hotmail.com

Recibido: 18 de enero de 2019

Aceptado para publicación: 18 de octubre de 2019 
underwent urinalysis and urine culture were collected. The following variables were considered: age, sex, reproductive status, breed, bodyweight, season, colour of urine, urine odour, urine density, $\mathrm{pH}$, glucose, proteins, blood, leukocytes, erythrocytes, crystals and type of crystals. In $60.8 \%$ (62/102) of the samples, bacteria were isolated in urine, including E. coli $40.3 \%$ (25/62), Klebsiella sp 12.9\% (8/62), Staphylococcus sp 12.9\% (8/62), Proteus sp $8.1 \%$ (5/62), Enterobacter sp 8.1\% (5/62) and Staphylococcus epidermidis $6.4 \%(4 / 62)$. Age, urinary density and presence of blood were statistically significant. In addition, it was determined that the urine density, the red colour of the urine, the presence of leukocytes and crystals represent risk factors $(p<0.05)$ for the occurrence of bacteriuria.

Key words: cats; urine culture; UTI; bacteriuria; FLUTD

\section{INTRODUCCIÓN}

La enfermedad del tracto urinario inferior felino (feline lower urinary tract disease - FLUTD), es el término que se usa para describir desórdenes que afectan la vejiga urinaria o la uretra de los gatos en las fases de almacenamiento y eliminación de la micción (Osborne et al., 2002; Westropp, 2013). El término FLUTD no es válido como diagnóstico pues se debe determinar la causa específica de los signos del tracto urinario inferior que presenta el paciente para recomendar el tratamiento apropiado (Little, 2014). Se ha reportado que FLUTD afecta entre 1.5 y $8 \%$ de los gatos atendidos (Lund et al., 1999; Lekcharoensuk et al., 2001), y de estos, las causas más reportadas son la urolitiasis que afectan alrededor del 15-20\% de los gatos con FLUTD, los problemas de conducta y defectos anatómicos pueden representar el 10\% de los casos, las neoplasias el 1-2\% y la infección del tracto urinario el 18\% (Kruger et al., 1991; Buffington et al., 1997; Gerber et al., 2005; Little, 2014).

Las infecciones del tracto urinario (ITU) son poco frecuentes en el gato; siendo las infecciones bacterianas las de mayor prevalencia (Davidson y Lees, 1993; Tabar y Planellas, 2010). La fisiopatología de la enfermedad está asociada al ascenso de bacterias por el tracto urinario y la colonización a los uréteres y riñones (Nelson y Couto, 2010; Senior, 2013). Los microorganismos de ma- yor frecuencia en ITU felinas son E. coli, Enterococcus sp, Proteus sp, Staphylococcus sp, Streptococcus sp, Klebsiela sp, Pseudomonas sp y Pasteurella sp (Davidson y Lees, 1993; Bailiff et al., 2006; Litster et al., 2007; Mayer-Roenne et al., 2007). Otros microorganismos que han sido aislados incluyen Enterobacter sp, Corynebacterium sp, Micrococcus sp y Propionibacterium sp (Bailiff et al., 2005; Puskar et al., 2007; Cavana et al., 2008). Los mecanismos de defensa del huésped están compuestos por micción normal, anatomía urinaria normal, barrera mucosa uroepitelial y propiedades antimicrobianas de la orina (Davidson y Lees, 1993).

La incidencia de ITU felina es variable (Eggertsdottir et al., 2007). Estudios retrospectivos en EEUU encontraron ITU en el $45 \%$ de gatos mayores de 10 años con signos de enfermedad urinaria inferior. Los gatos de edad avanzada pueden tener un mayor riesgo de ITU debido a la declinación de las defensas urinarias (Bartges, 2007). Así mismo, gatos con hipertiroidismo, diabetes mellitus y enfermedad renal crónica tienen mayor riesgo (Mayer-Roenne et al., 2007), así como animales de las raza Persa y Manx, las hembras y la pérdida de peso (Lekcharoensuk et al., 2001); no obstante, también puede presentarse en gatos con signos clínicos (Bailiff et al., 2006; Mayer-Roenne et al., 2007; Bartges, 2007; Litster et al., 2009; Little, 2014). 
El diagnóstico no debe basarse únicamente en el cuadro clínico, sino ser apoyado con los resultados del urianálisis completo y urocultivo. Además, en determinadas situaciones resulta útil la realización de pruebas de laboratorio adicionales, así como el empleo de técnicas de imagen que faciliten el diagnóstico de otras enfermedades asociadas (Cortadellas, 2010). La no detección de microorganismos en el sedimento no excluye su presencia ni descarta la ITU. El urocultivo es la prueba «gold» estándar para el diagnóstico de la ITU (Jarabo et al., 2018).

El objetivo del presente estudio retrospectivo fue describir los aislados bacterianos, así como la determinación de los factores de riesgo involucrados en la presentación de FLUTD.

\section{Materiales y Métodos}

El presente estudio retrospectivo fue transversal observacional descriptivo. El muestreo fue de tipo no probabilístico. Como criterios de inclusión se tomó en consideración historias clínicas de felinos que llegaron con manifestaciones clínicas de la enfermedad del tracto urinario inferior, en donde se encontró algún indicativo de presencia de bacteriuria en el análisis de orina y que posteriormente fue confirmado mediante urocultivo. Asimismo, se tomaron en consideración historias clínicas de felinos asintomáticos de enfermedad del tracto urinario inferior, en donde se tuvo un hallazgo incidental en los análisis de orina con algunos indicativos de la presencia de bacteriuria y que también fueron confirmados mediante un urocultivo.

El estudio se realizó en una clínica privada especializada en medicina felina en Lima, Perú. Se revisaron 2650 historias clínicas de pacientes felinos, de las cuales 1125 historias fueron casos de pacientes que presentaron enfermedad del tracto urinario inferior entre 2008 y 2015. De estas, 102 histo- rias que se ajustaron a los criterios de inclusión fueron seleccionadas. Se registraron datos del paciente (edad, sexo, estado reproductivo, raza, peso, estación del año) y datos del análisis de orina (color, olor, densidad urinaria, $\mathrm{pH}$, glucosa, proteínas, sangre, leucocitos, eritrocitos, cristales y tipo de cristales).

Los datos fueron analizados mediante la prueba de Chi cuadrado para establecer si presentaban asociación estadística significativa con bacteriurea $(\mathrm{p}<0.05)$. Además, se realizó un análisis de regresión logística múltiple de las variables en estudio, se establecieron los valores de Odds ratio y se determinaron los factores de riesgo para la presentación de bacteriuria con un intervalo de confianza de $95 \%$. Los datos fueron analizados con el paquete estadístico STATAv. 14.0.

\section{Resultados}

El $61.2 \%(62 / 102)$ de los urocultivos dio positivo y el $40.8 \%$ fue negativo. Los resultados para variables relacionadas con el paciente (edad, sexo, órganos reproductivos, raza y peso) se muestran en el Cuadro 1, en tanto que las para las variables relación con el examen de orina (físico, químico y de sedimento) se muestran en el Cuadro 2, incluyendo en ambos casos las asociaciones estadísticas $(\mathrm{p}<0.05)$.

Los factores de riesgo y los valores de Odds Ratio (OR) calculados para la presentación de bacteriuria se muestran en el Cuadro 3. La presentación de bacteriuria con la presencia de leucocitos ( $>5$ leucocitos/campo) muestra un riesgo de 6.25 veces mayor que la ausencia o presencia de menor cantidad de leucocitos ( $0-5$ leucocitos/campo) en el sedimento urinario. La presencia de cristales ( $>6$ cristales/campo) representa un riego 21.09 veces mayor que la presencia de d» 6 cristales/campo. Así mismo, una densidad urinaria d»1.035 representa un riesgo de 38.56 veces mayor con respecto a una mayor densidad urinaria $(>1.035)$. 
Cuadro 1. Frecuencia de bacteriuria y su asociación con potenciales factores de riesgo relacionados al paciente para la presentación de bacteriuria en 102 casos de pacientes con enfermedad del tracto urinario inferior felino (FLUTD) sintomático y asintomático

\begin{tabular}{llccccc}
\hline \multirow{2}{*}{ Variable } & & \multicolumn{2}{c}{ Total $(\mathrm{n})$} & \multicolumn{2}{c}{ Bacteriuria } & \multirow{2}{*}{ P value } \\
\cline { 2 - 5 } & & $\mathrm{n}$ & $\%$ & $\mathrm{n}$ & $\%$ & \\
\hline Edad (años) & $\leq 6$ & 65 & 63.7 & 34 & 54.8 & 0.024 \\
& $7-10$ & 20 & 19.6 & 13 & 21 & \\
\multirow{3}{*}{ Sexo } & $\geq 11$ & 17 & 16.7 & 15 & 24.2 & \\
& Macho & 69 & 67.7 & 41 & 66.1 & 0.683 \\
Órganos & Hembra & 33 & 32.4 & 21 & 33.9 & \\
reproductivos & Entero (a) & 39 & 38.2 & 24 & 38.7 & 0.902 \\
Raza & Castrado (a) & 63 & 61.8 & 38 & 61.3 & \\
& Mestizo & 78 & 76.5 & 46 & 74.2 & 0.5 \\
Peso (kg) & De raza & 24 & 23.5 & 16 & 25.8 & \\
& $<3$ & 11 & 10.8 & 10 & 16.1 & 0.064 \\
& $3-5$ & 54 & 52.9 & 28 & 45.2 & \\
& $>5-7$ & 31 & 30.4 & 19 & 30.7 & \\
& $>7$ & 6 & 5.9 & 5 & 8.1 & \\
\hline
\end{tabular}

La distribución porcentual de bacterias aisladas en el urocultivo se presenta en la Figura 1 , donde destaca la presencia de $E$. coli (40.3\%), seguido de Klebsiella sp (12.9\%) y Staphylococcus sp (12.9\%).

\section{Discusión}

La bacteria con mayor frecuencia de aislamiento en la orina de los gatos fue $E$. coli $(40.3 \%)(25 / 62)$. La mayoría de las infecciones del tracto urinario es causada por flora bacteriana derivada del tracto gastrointestinal o cutáneo, que cruzan el perineo y colonizan los genitales externos, para luego invadir la uretra y la vejiga en forma retrograda, en contra el flujo urinario (Nelson y Couto, 2010; Senior, 2013). Diversos estudios reportan a $E$. coli como el microorganismo aislado con mayor frecuencia en gatos y que puede representar un potencial zoonotico (Kurazono et al., 2003; Bailiff et al., 2006; Litster et al., 2007; Eggertsdottir et al., 2007; Mayer-Roenne et al., 2007). Otros microorganismos que han sido aislados incluyen Staphylococcus sp, Streptococcus sp, Enterococcus sp, Klebsiella sp, Proteus sp, Pseudomonas sp, Pasteurella sp, Enterobacter sp, Myco-plasma sp y Corynebacterium sp (Davidson y Lees, 1993; Bailiff et al., 2005; Bartges, 2007; Puskar et al., 2007; Cavana et al., 2008), muchos de los cuales fueron igualmente aislados en el presente estudio. La ITU habitualmente implica un solo organismo, aunque en $20-30 \%$ de los casos puede tratarse de infecciones bacterianas mixtas (Nelson y Couto, 2010).

En el presente estudio se obtuvo asociación estadística significativa $(\mathrm{p}<0.05)$ entre bacteriuria con la edad de los gatos. En estudios retrospectivos realizados en las universidades de Georgia y Tennessee se en- 
Cuadro 2. Frecuencia de bacteriuria y su asociación con potenciales factores de riesgo para la presentación de bacteriuria en 102 casos de pacientes con enfermedad del tracto urinario inferior felino (FLUTD) sintomático y asintomático

\begin{tabular}{|c|c|c|c|c|c|c|c|}
\hline \multirow[b]{2}{*}{ 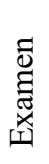 } & \multirow[b]{2}{*}{ Variable } & & \multicolumn{2}{|c|}{ Total (n) } & \multicolumn{2}{|c|}{ Bacteriuria } & \multirow[b]{2}{*}{$\begin{array}{c}\mathrm{P} \\
\text { value }\end{array}$} \\
\hline & & & $\mathrm{n}$ & $\%$ & $\mathrm{n}$ & $\%$ & \\
\hline \multirow{15}{*}{$\frac{.0}{\frac{0}{n}}$} & Estación & Verano & 19 & 18.6 & 10 & 16.1 & 0.338 \\
\hline & & Primavera & 31 & 30.4 & 20 & 32.3 & \\
\hline & & Invierno & 23 & 22.6 & 17 & 27.4 & \\
\hline & & Otoño & 29 & 28.4 & 15 & 24.2 & \\
\hline & Color & Amarillo claro & 19 & 18.6 & 12 & 19.4 & 0.161 \\
\hline & & Amarillo oscuro & 40 & 39.2 & 25 & 40.3 & \\
\hline & & Amarillo pálido & 7 & 6.9 & 7 & 11.3 & \\
\hline & & Marrón & 7 & 6.9 & 4 & 6.5 & \\
\hline & & Rojo & 29 & 28.4 & 14 & 22.6 & \\
\hline & Olor & Olor sui generis & 89 & 87.3 & 55 & 88.7 & 0.629 \\
\hline & & Olor amoniaco & 9 & 8.8 & 5 & 8.1 & \\
\hline & & Olor a acetona & 1 & 1 & 0 & 0 & \\
\hline & & Olor pútrido & 3 & 2.9 & 2 & 3.2 & \\
\hline & Densidad & $\leq 1.035$ (anormal) & 33 & 32.4 & 28 & 45.2 & 0.001 \\
\hline & & $>1.035$ (normal) & 69 & 67.7 & 34 & 54.8 & \\
\hline \multirow{18}{*}{ 异 } & $\mathrm{pH}$ & $>7.5$ (alcalino) & 88 & 86.3 & 54 & 87.1 & 0.764 \\
\hline & & 6-7.5 (normal) & 14 & 13.7 & 8 & 12.9 & \\
\hline & & $<6$ (ácido) & 0 & 0 & 0 & 0 & \\
\hline & Glucosa & Negativo & 81 & 79.4 & 50 & 80.7 & 0.293 \\
\hline & & $1+=50 \mathrm{mg} / \mathrm{dl}$ & 12 & 11.8 & 6 & 9.7 & \\
\hline & & $2+=100 \mathrm{mg} / \mathrm{dl}$ & 4 & 3.9 & 2 & 3.2 & \\
\hline & & $3+=300 \mathrm{mg} / \mathrm{dl}$ & 1 & 1 & 0 & 0 & \\
\hline & & $4+=1000 \mathrm{mg} / \mathrm{dl}$ & 4 & 3.9 & 4 & 6.5 & \\
\hline & Proteínas & Negativo & 11 & 10.8 & 8 & 12.9 & 0.063 \\
\hline & & Trazas & 5 & 4.9 & 2 & 3.2 & \\
\hline & & $1+=30 \mathrm{mg} / \mathrm{dl}$ & 32 & 31.4 & 24 & 38.7 & \\
\hline & & $2+=100 \mathrm{mg} / \mathrm{dl}$ & 17 & 16.7 & 6 & 9.7 & \\
\hline & & $3+=500 \mathrm{mg} / \mathrm{dl}$ & 37 & 36.3 & 22 & 35.5 & \\
\hline & Sangre & Negativo & 12 & 11.8 & 4 & 6.5 & 0.046 \\
\hline & oculta & $1+=10 \mathrm{Eri} / \mu 1$ & 7 & 6.9 & 6 & 9.7 & \\
\hline & & $2+=25 \mathrm{Eri} / \mu 1$ & 4 & 3.9 & 2 & 3.2 & \\
\hline & & $3+=50 \mathrm{Eri} / \mu \mathrm{l}$ & 6 & 5.9 & 6 & 9.7 & \\
\hline & & $4+=250 \mathrm{Eri} / \mu 1$ & 73 & 71.6 & 44 & 71.0 & \\
\hline \multirow{9}{*}{ 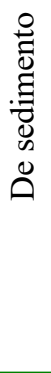 } & Leucocitos & 0-5/c (normal) & 62 & 60.8 & 33 & 53.2 & 0.052 \\
\hline & & $>5$ /c (anormal) & 40 & 39.2 & 29 & 46.8 & \\
\hline & Eritrocitos & 0-4/c (normal) & 35 & 34.3 & 23 & 37.1 & 0.461 \\
\hline & & $\geq 5 / c$ (anormal) & 67 & 65.7 & 39 & 62.9 & \\
\hline & Cristales & $\leq 6 / \mathrm{c}($ normal $)$ & 89 & 87.3 & 54 & 87.1 & 0.952 \\
\hline & & $>6 / \mathrm{c}$ (anormal) & 13 & 12.8 & 8 & 12.9 & \\
\hline & Tipos de & Ausencia & 50 & 49 & 34 & 54.8 & 0.3 \\
\hline & cristales & Estruvita & 49 & 48 & 26 & 41.9 & \\
\hline & & Oxalato de calcio & 3 & 2.9 & 2 & 3.2 & \\
\hline
\end{tabular}


Cuadro 3. Presentación de bacteriuria y su asociación con potenciales factores de riesgo en 102 casos de pacientes con enfermedad del tracto urinario inferior felino (FLUTD) sintomático y asintomático

\begin{tabular}{|c|c|c|c|}
\hline Factor de riesgo & Odds ratio & $\mathrm{P}>|\mathrm{z}|$ & $\begin{array}{l}\text { Intervalo de Confidencia } \\
\qquad(95 \%)\end{array}$ \\
\hline \multicolumn{4}{|l|}{ Edad $\leq 6$ años } \\
\hline $7-10$ & 4.28 & 0.15 & $0.57-32.14$ \\
\hline$\geq 11$ & 10.32 & 0.16 & $0.38-273.99$ \\
\hline \multicolumn{4}{|l|}{ Sexo macho } \\
\hline Macho / Hembra & 0.25 & 0.12 & $0.04-1.43$ \\
\hline \multicolumn{4}{|l|}{ Órganos reproductivos } \\
\hline Entero / Castrado & 2.02 & 0.42 & $0.35-11.49$ \\
\hline \multicolumn{4}{|l|}{ Raza mestizo } \\
\hline \multirow{2}{*}{\multicolumn{4}{|c|}{ Peso $<3 \mathrm{~kg}$}} \\
\hline & & & \\
\hline $3-5 \mathrm{~kg}$ & 0.05 & 0.06 & $0.002-1.15$ \\
\hline$>5-7 \mathrm{~kg}$ & 0.2 & 0.34 & $0.007-5.42$ \\
\hline$>7 \mathrm{~kg}$ & 0.6 & 0.83 & $0.005-68.14$ \\
\hline \multicolumn{4}{|l|}{ Estación verano } \\
\hline Primavera & 0.48 & 0.54 & $0.049-4.83$ \\
\hline Invierno & 3.51 & 0.24 & $0.41-29.42$ \\
\hline Otoño & 0.1 & 0.06 & $0.01-1.14$ \\
\hline \multicolumn{4}{|l|}{ Orina color amarillo claro } \\
\hline Amarillo oscuro & 0.22 & 0.14 & $0.03-1.63$ \\
\hline Marrón & 0.04 & 0.09 & $0.001-1.64$ \\
\hline Rojo & 0.07 & 0.04 & $0.006-0.96$ \\
\hline \multicolumn{4}{|l|}{ Orina olor amoniaco } \\
\hline Amoniaco & 1.29 & 0.84 & $0.09-17.24$ \\
\hline Pútrido & 1.47 & 0.85 & $0.02-90.91$ \\
\hline \multicolumn{4}{|l|}{ Glucosa negativo (0) } \\
\hline $50 \mathrm{mg} / \mathrm{dl}$ & 0.07 & 0.06 & $0.005-1.12$ \\
\hline $100 \mathrm{mg} / \mathrm{dl}$ & 0.05 & 0.12 & $0.001-2.12$ \\
\hline \multicolumn{4}{|l|}{ Proteínas negativo $(0)$} \\
\hline $30 \mathrm{mg} / \mathrm{dl}$ & 13.99 & 0.05 & $0.92-211.3$ \\
\hline $100 \mathrm{mg} / \mathrm{dl}$ & 0.99 & 0.99 & $0.059-16.778$ \\
\hline $500 \mathrm{mg} / \mathrm{dl}$ & 2.87 & 0.4 & $0.23-35.18$ \\
\hline \multicolumn{4}{|l|}{ Sangre negativo $(0)$} \\
\hline 10 eritrocitos $/ \mu 1$ & 1.47 & 0.79 & $0.075-29.08$ \\
\hline 25 eritrocitos $/ \mu 1$ & 0.03 & 0.37 & $0.00001-57.94$ \\
\hline 250 eritrocitos $/ \mu 1$ & 0.34 & 0.43 & $0.022-5.13$ \\
\hline \multicolumn{4}{|l|}{ pH 6.0-7.5 } \\
\hline \multirow[b]{2}{*}{ Leucocitos $0-5 / \mathrm{c}$} & 0.33 & 0.37 & $0.029-3.75$ \\
\hline & 6.25 & 0.04 & $1.04-37.58$ \\
\hline \multicolumn{4}{|l|}{ Eritrocitos $0-4 / \mathrm{c}$} \\
\hline & 0.49 & 0.52 & $0.059-4.04$ \\
\hline \multicolumn{4}{|l|}{ Cristales $<6 / \mathrm{c}$} \\
\hline \multicolumn{4}{|l|}{ Densidad urinaria $>1.035$} \\
\hline & 38.56 & 0.007 & $2.72-545.77$ \\
\hline Constante & 868.47 & 0.01 & $5-150682$ \\
\hline
\end{tabular}




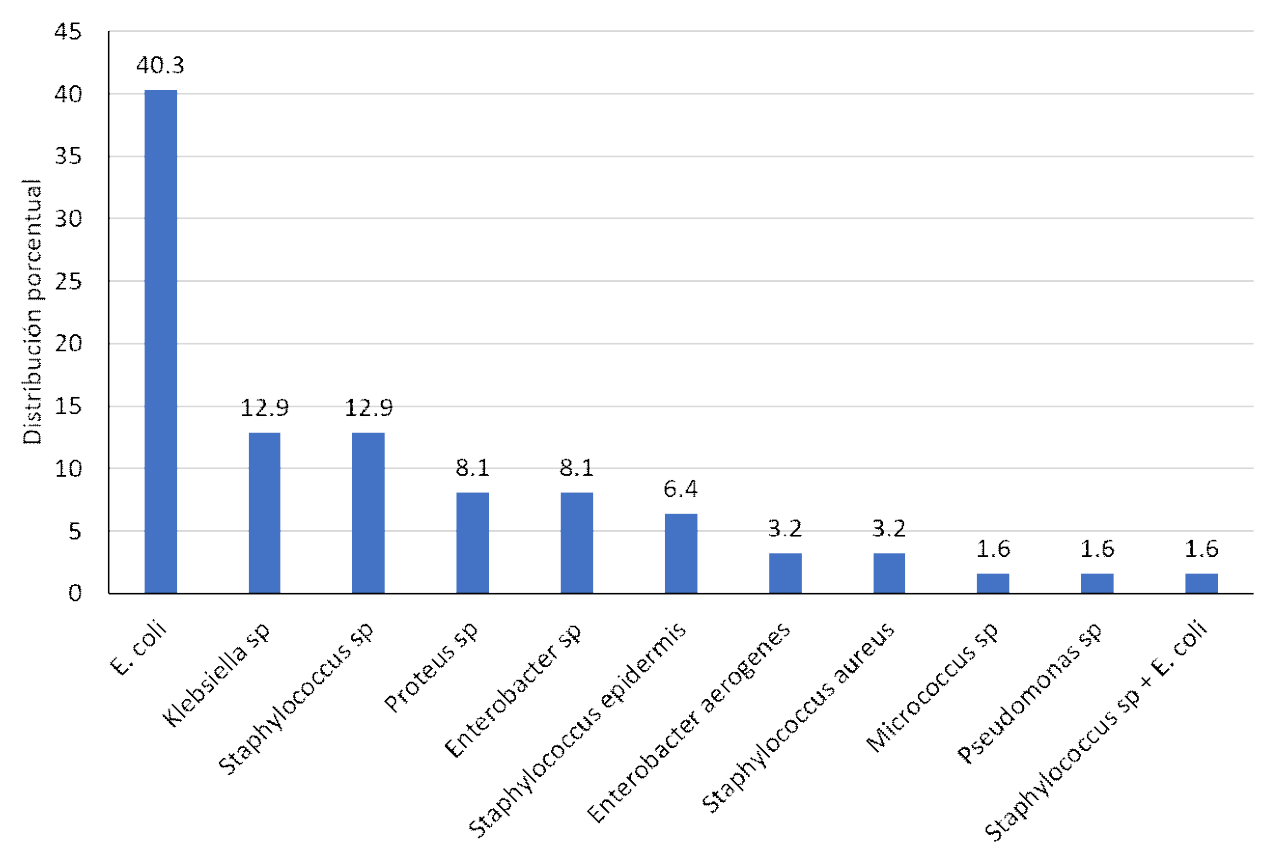

Figura 1. Distribución porcentual de bacteriuria en urocultivos realizados durante el periodo 20082015 en pacientes felinos con enfermedad del tracto urinario inferior felino (FLUDT) sintomático y asintomático

contró que el $45 \%$ de los casos de gatos con signos de enfermedad urinaria inferior se presentaron en gatos mayores de 10 años (Bartges, 2007). Las observaciones de esta casuística indica que la ITU es poco común en gatos jóvenes con enfermedad urinaria inferior, pero que es un problema importante en animales gerontes (Lekcharoensuk et al., 2001).

Se observó asociación estadística significativa entre bacteriuria y la densidad urinaria $(\mathrm{p}<0.05)$. El 54.8\% (34/62) de los gatos con bacteriuria tuvieron una densidad normal $(>1.035)$. En condiciones normales, la orina de los gatos es más concentrada que la del perro, lo cual es uno de los motivos para la escasa incidencia de ITU en gatos normales (Bartges y Barsanti, 2001; Nelson y Couto, 2010). La orina diluida formada en animales con procesos poliúricos/polidípsicos tiene menos propiedades antibacterianas que las orinas hiperstenúricas (Nelson y Couto, 2010; Senior, 2013). Algunos estudios han reportado que la densidad urinaria es significativamente más baja en gatos con ITU inferior (Litster et al., 2009), lo cual podría estar relacionado con insuficiencia renal, diabetes mellitus, hipertiroidismo o terapéutica con diuréticos (Bartges et al., 1996; Bartges, 2007; Nelson y Couto, 2010).

El $71.0(44 / 62)$ de casos de bacteriourea presentaron sangre oculta en orina en el nivel de $4+(250$ eritrocitos/ $\mu 1)$. El hallazgo de glóbulos rojos en el sedimento confirma la hematuria. La orina muy diluida o alcalina puede causar lisis de los eritrocitos y estos no son siempre visibles en el sedimento (Scherk, 2014); a pesar de ello, en el presente estudio la sangre en orina no resultó un factor de riesgo para la presentación de bacteriuria. 
Si bien no hubo diferencias significativas en animales con bacteriuria frente a la mayor o menor cantidad de leucocitos, eritrocitos, proteínas y glucosa en la orina, la sola presencia de leucocitos en el sedimento de la orina es un indicador de inflamación o infección (Rubio, 2011). Si bien es cierto, no hubo asociación estadística significativa con le presencia de leucocitos en orina, estos resultaron ser un factor de riesgo para la presentación de bacteriuria $(\mathrm{p}<0.05)$.

\section{Conclusiones}

- Las bacterias más frecuentemente aisladas de pacientes con enfermedad del tracto urinario inferior felino (FLUTD) sintomático y asintomático fueron $E$. coli (40.3\%, 25/62), Klebsiella sp (12.9\%) y Staphylococcus sp (12.9\%).

- Se encontró asociación significativa $(\mathrm{p}<0.05)$ entre la presentación de bacteriuria y las variables edad, densidad urinaria y presencia de sangre oculta.

- Los factores de riesgo para la presentación de bacteriuria en pacientes con FLUTD sintomático y asintomático fueron densidad urinaria, presencia de leucocitos y cristales en el sedimento urinario, y color rojo de la orina.

\section{Literatura Citada}

1. Bailiff N, Nelson R, Feldman E, Westropp J, Ling G, Jang S, Kass P. 2006. Frequency and risk factors for urinary tract infection in cats with diabetes mellitus. J Vet Intern Med 20: 850855. doi: 10.1892/0891-6640(2006)20[850:-farffu $] 2.0 . \mathrm{co} ; 2$

2. Bailiff NL, Westropp J, Jang S, Ling BG. 2005. Corynebacterium urealyticum urinary tract infection in dogs and cats: 7 cases (1996-2003). J Am Vet Med Assoc 226:1676-1680. doi: 10.2460/ javma.2005.226.1676
3. Bartges J, Barsanti J. 2001. Infección bacteriana de vías urinarias en gatos. En: Bonagura J (ed). Terapéutica veterinaria de pequeños animales. Vol 2. España: McGraw-Hill-Interamericana. p 939-945.

4. Bartges J, Finco R, Polzin D, Osborne C, Barsanti J, Brown S. 1996. Pathophysiology of urethral obstruction. Vet Clin N Am-Small 26: 255-264. doi: 10.1016/S0195-5616(96)50206-8

5. Bartges J. 2007. Revisión de la infección urinaria bacteriana. En: August J (ed). Consultas en medicina felina. Argentina: Inter-Médica. p 453-458.

6. Buffington $C$, Chew D, Kendall $M$, Scrivani P, Thompson S, Blaisdell J, Woodworth B. 1997. Clinical evaluation of cats with nonobstructive urinary tract diseases. J Am Vet Med Assoc 210: 46-50.

7. Cavana P, Zanatta R, Nebbia P, Miniscalco B, Vittone V, Zanoni MG, Serra $R$, et al. 2008. Corynebacterium urealyticum urinary tract infection in a cat with urethral obstruction. J Feline Med Surg 10: 269-273. doi: 10.1016/ j.jfms.2007.12.003

8. Cortadellas O. 2010. Tratamiento de las infecciones del tracto urinario. En: Manual de nefrología y urología clínica canina y felina. Zaragoza: Servet. p 239$246 \mathrm{p}$.

9. Davidson A, Lees G. 1993. Diagnóstico y tratamiento de la uropatía infecciosa. En: August J (ed). Consultas en medicina felina. Buenos Aires: Inter-Médica. p. 343-349.

10. Eggertsdottir A, Lund H, Krontveit R, Sorum H. 2007. Bacteriuria in cats with feline lower urinary tract disease: a clinic study of 134 cases in Norway. J Feline Med Surg 9:458-65. doi: 10.1016/ j.jfms.2007.06.003

11. Gerber B, Boretti F, Kley S, Laluha P, Müller C, Sieber N, Unterer $S$, et al. 2005. Evaluation of clinical signs and causes of lower urinary tract disease in European cats. J Small Anim Pract 46: 571-577. doi: 10.1111/j.1748-5827.2005.tb00288.x 
12. Jarabo M, Asencio M, Carranza R, Herráez O, Huertas M, et al. 2018. Proyecto URISCAM: Evaluación multicéntrica del citómetro UF-Series en el despistaje de infecciones urinarias. Rev Esp Quimioter 31(1): 13-20.

13. Kruger JM, Osborne CA, Goyal SM, Wickstrom SL, Johnston GR, Fletcher TF, Brown PA. 1991. Clinical evaluation of cats with lower urinary tract disease. J Am Vet Med Assoc 199: 211-216.

14. Kurazono H, Nakano M, Yamamoto $S$, Ogawa O, Yuri K, Nakata K, Kimura M, et al. 2003. Distribution of the usp gene urophatognic Escherichia coli isolated from companion animals and correlation with serotypes and sizevariations of the pathogenicity island. Microbiol Immunol 47: 797-802. doi: 10.1111/j.1348-0421.2003.tb03437.x

15. Lekcharoensuk C, Osborne C, Lulich J. 2001. Epidemiologic study of risk factors for lower urinary tract diseases in cats. J Am Vet Med Assoc 218: 14291445. doi: 10.2460/javma.2001.218.1429

16. Litster A, Moss S, Platell J, Trott DJ. 2009. Occult bacterial lower urinary tract infections in cats-urinalysis and culture findings. Vet Microbiol 136: 130134. doi: 10.1016/j.vetmic.2008.10.019

17. Litster A, Moss SM, Honnery M, Rees B, Trott DJ. 2007. Prevalence of bacterial species in cats with clinical signs of lower urinary tract disease: recognition of Staphylococcus felis as a possible feline urinary tract pathogen. Vet Microbiol 121: 182-188. doi: 10.1016/ j.vetmic.2006.11.025

18. Little S. 2014. Tracto urinario inferior. El gato, medicina clínica y tratamiento. Argentina: Inter-Médica. p. 1676.

19. Lund EM, Armstrong PJ, Kirk CA, Kolar LM, Klausner JS. 1999. Health status and population characteristics of dogs and cats examined at private veterinary practices in the United States. J Am Vet Med Assoc 214: 1336-1341.
20. Mayer-Roenne B, Goldstein R, Erb $H N$. 2007. Urinary tract infections in cats with hyperthyroidism, diabetes mellitus and chronic kidney disease. $\mathrm{J}$ Feline Med Surg 9: 124-32. doi: 10.1016/ j.jfms.2006.09.004

21. Nelson R, Couto G 2010. Enfermedad del aparato urinario inferior felino. En: Medicina interna de pequeños animales. $4^{\mathrm{a}}$ ed. Barcelona: Elsevier. p 677-683.

22. Osborne C, Kruger J, Lulich J, Polzin D, Lekcharoensuk C. 2002. Enfermedades urinarias inferiores felinas. En: Ettinger S, Feldman E (eds). Tratado de medicina interna veterinaria. Argentina: Inter-Médica. p 1905-1946.

23. Puskar M, Lemons C, Papich MG, Vaden SL, Birkenheuer A. 2007. Antibiotic-resistant Corynebacterium jeikeium urinary tract infection in a cat. J Am Anim Hosp Assoc 43: 61-64. doi: 10.5326/0430061

24. Rubio A. 2011. Actualización de la enfermedad del tracto urinario inferior. En: Minovich F, Paludi A (eds). Medicina felina práctica. $3^{a}$ ed. España: Multimédica. p 405-425.

25. Scherk M. 2014. Enfermedad del tracto urinario. En: Little $S$ (ed). El gato: medicina clínica y tratamiento. Argentina: Inter-Médica. p 1128-1224.

26. Senior D. 2013. Infección del tracto urinario: bacterias. En: Bartges J, Polzin D (eds). Nefrología y urología de pequeños animales. Argentina: Inter-Médica. p 728-734.

27. Tabar M, Planellas M. 2010. Enfermedad del tracto urinario inferior felino. En: Cortadellas O (ed). Manual de nefrología y urología clínica canina y felina. España: Servet. p 223-238.

28. Vail DM, Allen TA, Weiser G. 1986. Applicability of leukocyte esterase test strip in detection of canine pyuria. J Am Vet Med Assoc 189: 1451-1453.

29. Westropp J. 2013. Cistitis idiopática felina. En: Bartges J, Polzin D (eds). Nefrología y urología de pequeños animales. Argentina: Inter-Médica. p 765-774. 